\title{
Performance of the Tile PreProcessor Demonstrator for the ATLAS Tile Calorimeter Phase II Upgrade
}

\author{
F. Carrión ${ }^{a},{ }^{*}$ P. Moreno ${ }^{a}$ and A. Valero ${ }^{a}$, on behalf of the ATLAS Tile Calorimeter \\ System \\ ${ }^{a}$ Instituto de Física Corpuscular (CSIC-Universidad de Valencia), \\ Catedrático José Beltrán 2, Paterna, Spain. \\ E-mail: fernando.carrio@cern.ch
}

\begin{abstract}
The Tile Calorimeter PreProcessor demonstrator is a high performance double AMC board based on FPGA resources and QSFP modules. This board has been designed in the framework of the ATLAS Tile Calorimeter Demonstrator Project for the Phase II Upgrade as the first stage of the back-end electronics. The TilePPr demonstrator has been conceived for receiving and processing the data coming from the front-end electronics of the TileCal Demonstrator module, as well as for configuring it. Moreover, the TilePPr demonstrator handles the communication with the Detector Control System to monitor and control the front-end electronics. The TilePPr demonstrator represents $1 / 8$ of the final TilePPr that will be designed and installed into the detector for the ATLAS Phase II Upgrade.
\end{abstract}

KEYWORDS: ATLAS; Tile Calorimeter; Phase II Upgrade; TilePPr; FPGA; Optical modules.

\footnotetext{
* Corresponding author.
} 


\section{Contents}

1. Introduction 1

1.1 Current architecture 2

1.2 Phase II Upgrade architecture 2

1.3 Tile Calorimeter Demonstrator Project 3

2. The TilePPr prototype 3

2.1 Hardware 4

2.2 Firmware 5

3. Status 6

$\begin{array}{ll}\text { 4. Conclusions } & 8\end{array}$

$\begin{array}{ll}\text { References } & 8\end{array}$

\section{Introduction}

ATLAS (A Toroidal LHC ApparatuS) [1] is a general purpose detector designed to explore the products of the proton-proton and heavy ions collisions from the Large Hadron Collider (LHC) at CERN in Geneva, Switzerland. The Hadronic Tile Calorimeter (TileCal) detector is one of the several subsystems composing the ATLAS experiment which covers the central region. TileCal [2] is a sampling calorimeter made out of steel plates and plastic scintillator tiles which is divided in three cylindrical parts: a central long barrel (LBA, LBC) and two extended barrels (EBA, EBC) (Figure 1). Each cylindrical part is formed by 64 wedges which are divided in cells, and each cell is read out using two photomultipliers (PMTs) and wave length shifting fibers. A total of 9852 PMTs are needed for the complete readout of TileCal.

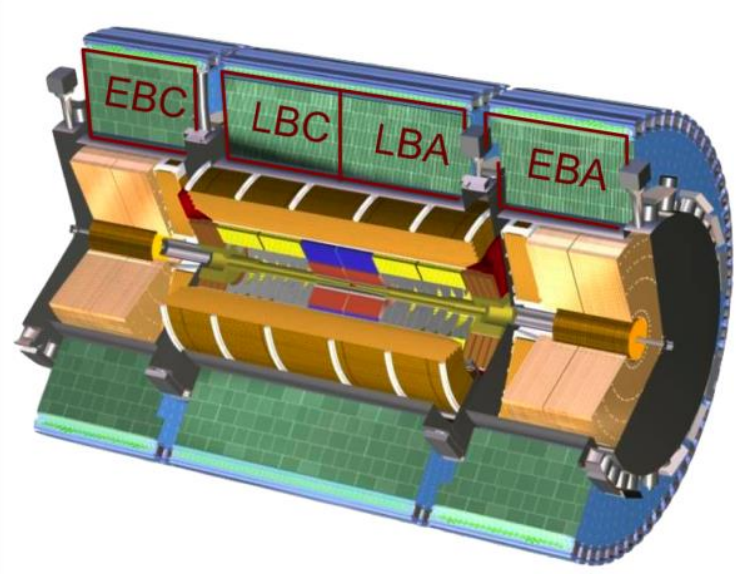

Figure 1. Drawing of the TileCal detector. 
After the Phase II upgrade, the High Luminosity Large Hadron Collider (HL-LHC) will provide to ATLAS [3] and CMS an instantaneous luminosity around $5 \times 10^{34} \mathrm{~cm}^{-2} \mathrm{~s}^{-1}$ with a pileup close to 200 events and will deliver a total integrated luminosity of $3000 \mathrm{fb}^{-1}$ in ten years. The new requirements for the ATLAS Phase II impose a complete upgrade of the current readout architecture and trigger systems of the TileCal to improve the event selections and cope with the new radiation levels, latency and high data rates.

\subsection{Current architecture}

In the current system, all the front-end readout electronics are placed at the outer radius of the detector inside long drawers called super-drawers. Each super-drawer contains all the electronics needed to read out one TileCal module (up to 48 PMTs).

Figure 2 shows a block diagram of the current readout architecture. The PMTs signals are shaped and amplified in a parallel bi-gain chain with a nominal ratio of 1:64. Low gain signals are analog summed to form towers of cells and sent to the Level-1 Calorimeter trigger system (L1Calo) for trigger decision. The shaped signals are digitized at the bunch crossing frequency (every $\sim 25 \mathrm{~ns}$ ) and stored in pipelined buffers in the Digitizer boards. When a trigger signal (Level-1 trigger acceptance, L1A) from the L1Calo is received, the Digitizer extracts the selected event from the pipelined buffers and the Interface board gathers and transmits the digitized event to the back-end electronics at a maximum average frequency of $100 \mathrm{kHz}$. The Read-Out Drivers (ROD) [4] perform data processing tasks in real time sending the results to the Read-Out Buffers (ROB) in the next trigger level.

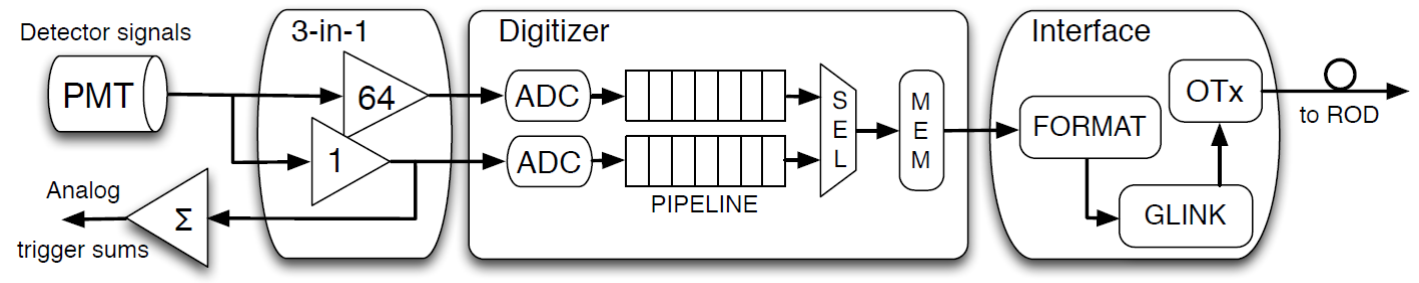

Figure 2. Diagram of the current readout architecture of the Tile Calorimeter.

\subsection{Phase II Upgrade architecture}

The new readout architecture proposed for the Tile Calorimeter points to a full digital trigger system with full precision and granularity to improve the event selection. In order to operate in the higher radiation environment, the new readout electronics system will include redundant optical fibers between the front-end and back-end electronics, radiation-tolerant electronics, redundant low voltage power supplies and will use for data transmission more reliable protocols with error correction.

Figure 3 shows a block diagram of the proposed readout for the Phase II Upgrade. In this new readout architecture the DaughterBoard [5] in the front-end electronics will transmit the digitized signals from the PMTs to the Tile PreProcessor (TilePPr) [6] in the back-end electronics for every bunch crossing ( $25 \mathrm{~ns}$ ), leading into a significant increase of the data rate. The TilePPr will store the digitized samples until the reception of a trigger acceptance signal when the data will be transmitted to the Front End LInk eXchange [7] (FELIX) system. Table I shows a comparison between the data bandwidth of the current system and the Phase II 
architecture. The TilePPr will transmit preprocessed data to the first level of trigger with improved precision and granularity for trigger decision.

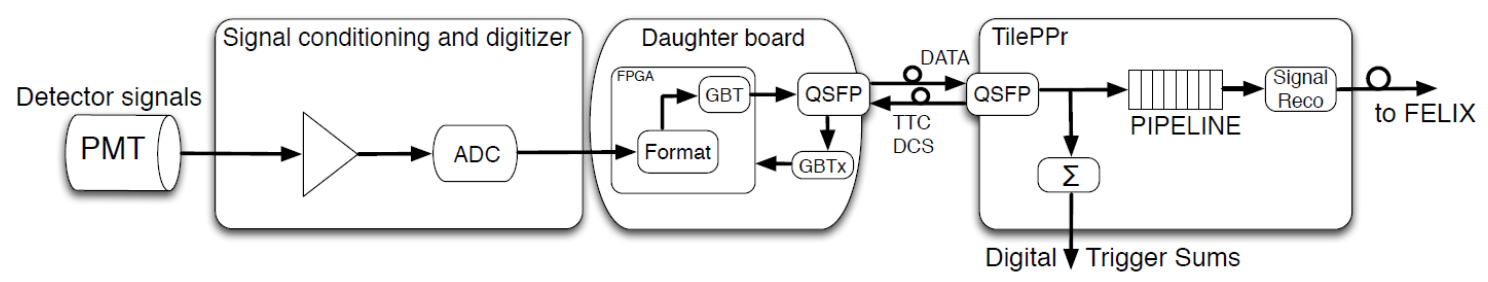

Figure 3. Diagram of the Phase II readout architecture of the Tile Calorimeter.

\begin{tabular}{ccc} 
& Present & Phase II \\
\hline Total BW & $\sim 205 \mathrm{Gbps}$ & $\sim 80 \mathrm{Tbps}$ \\
N. fibers & 256 & 8192 \\
BW / module & $800 \mathrm{Mbps}$ & $320 \mathrm{Gbps}$ \\
Nb. boards & 32 & 32 \\
Nb. crates & $4(\mathrm{VME})$ & 4 (ATCA) \\
In BW/board & $6.4 \mathrm{Gbps}$ & $2.5 \mathrm{Tbps}$ \\
Out BW/board & $2.56 \mathrm{Gbps}$ & $40 \mathrm{Gbps}$ \\
Out BW/board $_{\mathrm{LA} / \mathrm{LO}}$ & Analog & $500 \mathrm{Gbps}$
\end{tabular}

Table 1. Comparison between the data requirements in the present and the Phase II proposed architecture.

\subsection{Tile Calorimeter Demonstrator Project}

The Demonstrator project aims to validate on a smaller scale the new readout architecture and trigger system interfaces before the complete installation of the upgraded electronics in ATLAS.

The Demonstrator project plans include test beam campaigns for the evaluation of the front-end electronics during 2015 and at the end of 2016, the installation into the ATLAS detector of a Demonstrator slice with the upgraded electronics. Since the full digital trigger system will not be present until Phase II, the Demonstrator module will also provide analog trigger signals to the current L1Calo system.

The Demonstrator module comprises on a single frame, the on-detector electronics needed for the digitization of signals coming from the PMTs, the calibration circuitry and high speed data communication with the back-end electronics, and the electronics needed for the distribution and monitoring of high voltage to the PMTs.

\section{The TilePPr prototype}

The TilePPr prototype is the main component of the back-end electronics in the Demonstrator project, providing compatibility between the system and the upgraded system. The prototype described in this note can operate one complete TileCal module. The TilePPr receives and process digital data from the TileCal module, and also decodes and distributes Trigger Timing and Control (TTC) signals to the front-end electronics for configuration and synchronization with the LHC clock. The TilePPr prototype interfaces the Detector Control System (DCS) for controlling and monitoring the high voltage power supplies in the front-end electronics. This prototype serves also as a test-bench for the development of new algorithms for the 
reconstruction of energy, time and quality factor to be used in the final system after Phase II Upgrade.

\subsection{Hardware}

The TilePPr has been designed as a double mid-size Advanced Mezzanine Card (AMC) [8] form factor as shown in Figure 4. This board can be operated in an Advanced Telecommunications Computing Architecture (ATCA) carrier or in a Micro Telecommunications Computing Architecture $(\mu \mathrm{TCA})$ crate.

The stack-up has been designed to fulfil the high-speed design specifications and constraints. NELCO 4000-13 SI has been selected as dielectric material in order to obtain low losses at high frequency. The PCB counts 16 layers, divided in 8 layers for power and ground planes and 8 layers for signals with a total thickness of the PCB of $1.6 \mathrm{~mm}$, compliant with the AMC standard.

The core processing of the TilePPr prototype is composed by two Xilinx series 7 FPGAs [9]: one Virtex 7 (XC7VX485T) and one Kintex 7 (XC7K420T). These FPGAs provide high connectivity bandwidth, high-density logic resources and dedicated Digital Signal Processors slices (DSP) resources for the implementation of signal processing algorithms.

The Virtex 7 FPGA is connected to 4 QSFP+ providing a high speed path with the frontend electronics at a maximum data rate of 160 Gbps using the embedded GTX transceivers. This FPGA is also connected to an Avago MiniPOD receiver for testing purposes and to six high speed lines of an Avago MiniPOD transmitter for the communication with the upgraded trigger system. The Kintex 7 FPGA is connected to the rest of the high speed lines of the Avago MiniPOD transmitter to evaluate other options for the communication with the upgraded trigger system.

DDR3 memories connected to both FPGAs enhance the flexibility of the design. These memories are envisaged for the integration of embedded operating system such as light embedded Linux and for storing event samples.

A Modular Management Controller (MMC) board [10] provides the operation of the basic services to the prototype when connected to the ATCA system using the Intelligent Platform Management Interface (IPMI) protocol. The MMC manages the power connection of the TilePPr with the carrier and monitors the temperature sensors on the board.

A third FPGA, one Xilinx Spartan 6, is present in the design for slow control purposes and monitoring of the jitter cleaners, clock management units, QSFP and MiniPOD modules and supervisory power chips. The Spartan 6 is connected to the MMC through an $\mathrm{I}^{2} \mathrm{C}$ module allowing remote access to the TilePPr through IPMI.

In order to expand the functionality of the prototype, the TilePPr includes a High Pin Count FPGA Mezzanine Card (HPC FMC) and other physical communications as USB-UART interfaces or Ethernet for debugging. The AMC connector provides high speed communication with the ATCA system through the $5 \mathrm{GbE}$ and 1 PCIe ports. 


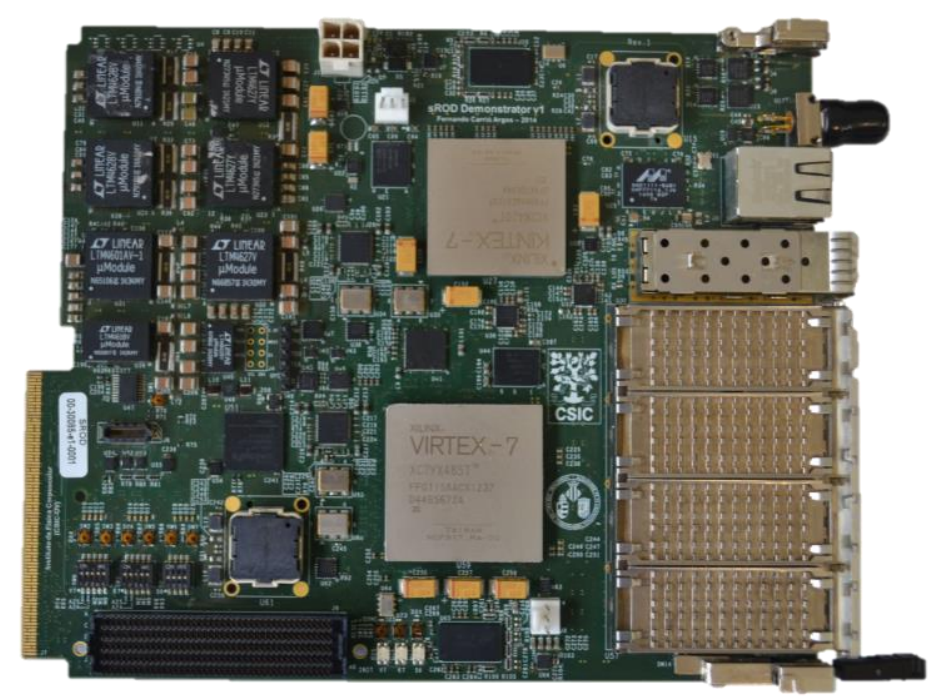

Figure 4. First prototype of the TilePPr.

\subsection{Firmware}

The Virtex 7 operates the TileCal module through four QSFPs using 16 asymmetric GigaBit Transceiver (GBT) [11] running at $4.8 \mathrm{Gbps}$ for the downlink (TilePPr to front-end) and 9.6 Gbps for the uplink (front-end to TilePPr).

DCS commands and TTC information are encoded and transmitted to the downlink at a data rate of 4.8 Gbps using the standard GBT protocol with Forward Error Correction (FEC). In the front-end electronics the DaugtherBoard decodes and executes the received command, sending an acknowledgement receipt to the TilePPr.

The readout data and monitoring are received and decoded using the GBT protocol at 9.6 Gbps. For the uplink the FEC capability has been disabled in order to increase the standard GBT payload, while a 16-bit Cyclic Redundancy Check (CRC) is used for error detection.

The firmware implemented in the Virtex 7 FPGA includes modules to unpack the received data frame, perform CRC checking and store event samples in configurable-delay pipelines. High and low gain samples are sent for every bunch crossing ( $25 \mathrm{~ns})$.

Once the samples have been extracted from the incoming data frame, events are stored in circular pipeline memories with a $12,5 \mu$ s depth implemented in $18 \mathrm{~Kb}$ block RAMs. When the L1A signal is received, the selected events are copied to the de-randomizers memories and then packed and transmitted to the present RODs through an SFP using the G-Link protocol. In that way the TilePPr prototype allows backward compatibility with the current readout architecture.

TTC commands are decoded through a dedicated IP core which interfaces with a GTX transceiver. The TTC clock is extracted and cleaned using an external jitter cleaner chip to reduce the jitter levels up to the required values for the GTX transmission. In order to work in the same clock domain, the DaughterBoard in the front-end electronics recovers the TTC clock from the incoming data and uses it as clock reference for the transmission to the TilePPr prototype, achieving synchronous communication between the front-end and the TilePPr.

All the data flow and control of the TilePPr is implemented over an Ethernet network using the IPbus protocol [12]. A set of IPbus registers are used to handle the TTC and DCS 
commands to the front-end as well as for data taking purposes. Configuration and remote monitoring of the TilePPr is also managed through the IPbus registers.

Figure 5 shows a block diagram of the firmware described in this section.

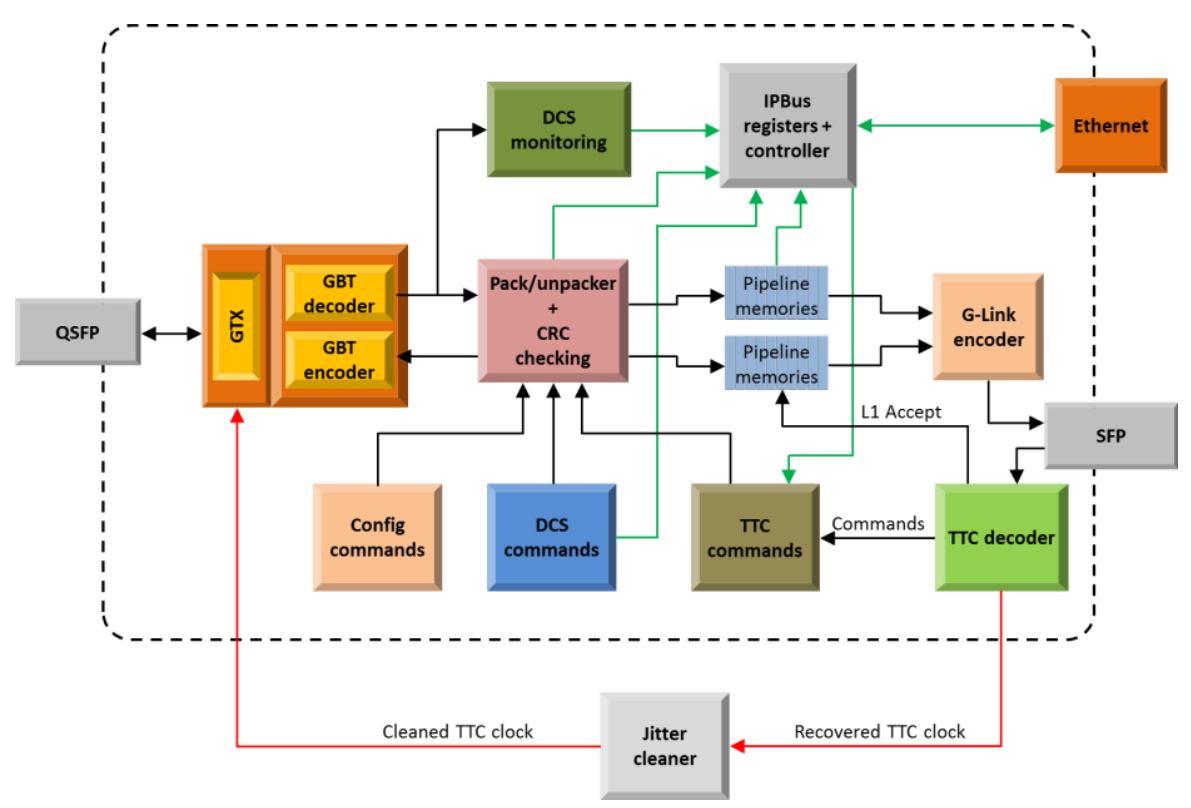

Figure 5. Block diagram of the TilePPr prototype firmware.

Table 2 lists a summary of the Virtex 7 resources occupied with the implementation of the firmware described in this section.

\begin{tabular}{lccc}
\multicolumn{1}{c}{ Resources } & Used & Available & Utilization \\
\hline Slice Registers & 144,747 & 607,200 & $23 \%$ \\
Slice LUTs & 145,128 & 303,600 & $47 \%$ \\
RAMB36E1 & 283 & 1,030 & $27 \%$ \\
RAMB18E1 & 328 & 2,060 & $15 \%$ \\
Global Clock Buffers & 29 & 32 & $90 \%$ \\
Horizontal Clock Buffers & 23 & 168 & $13 \%$ \\
MMCMs & 10 & 14 & $71 \%$ \\
PLLs & 5 & 14 & $35 \%$ \\
GTX MGTs & 18 & 48 & $38 \%$
\end{tabular}

Table 2. Virtex 7 resource utilization.

\section{Status}

The first TilePPr prototypes have been designed, produced and tested as part of the TileCal Demonstrator project. The Xilinx IBERT IP core has been implemented for the validation of the signal integrity of the QSFP lines using an external optical loopback. During the BER tests, no errors were observed on sixteen links running at 9.6 Gbps with a PRBS-31 data pattern over a period of 115 hours, which corresponds to a BER $\leq 5 \cdot 10^{-17}$ with a confidence level of $95 \%$. Figure 6 shows the eye diagram obtained using the IBERT IP core on the GTX transceivers used for the uplink and downlink communication paths. 

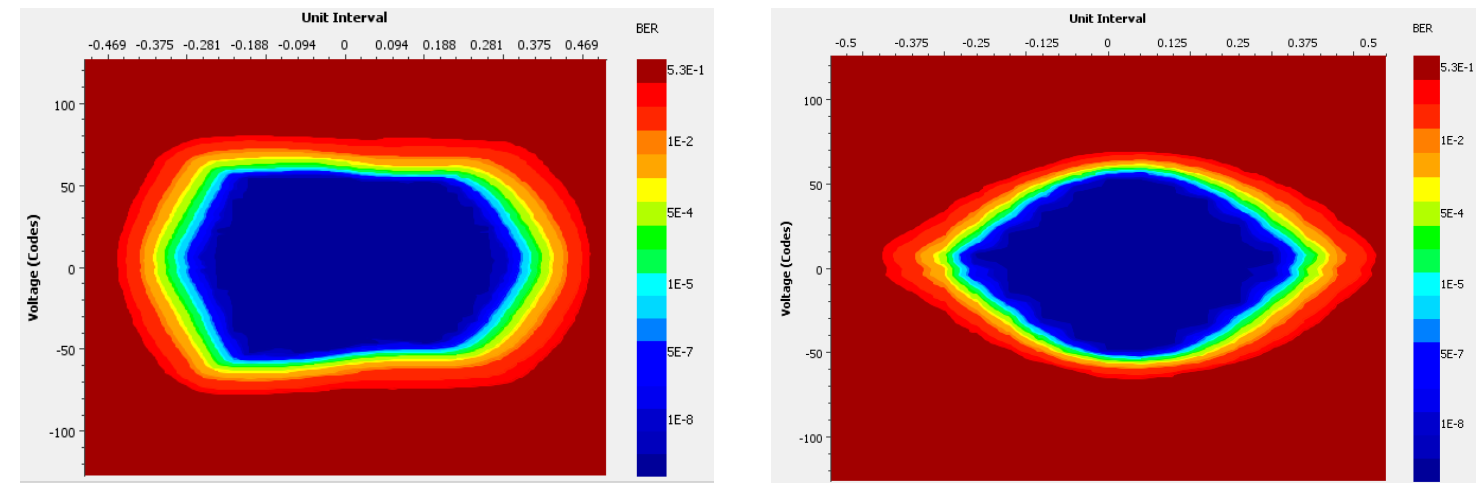

Figure 6. (a) Eye diagram at 4.8 Gbps (b) Eye diagram at 9.6 Gbps.

A series of $\mathrm{C}++/$ Python scripts allows remote operation of the TilePPr through the GbE ports using the IPbus protocol for monitoring, data taking and calibration. Different tests are now available as pedestal stability, pedestal linearity, high voltage stability or calibration tests using charge injection pulses.

The results of a charge injection linearity test in a typical channel are presented in the profile histogram showed in Figure 7. These results show a good linearity between the programmed injected charge (Inj Q) in the front-end electronics and the reconstructed charge in the TilePPr (Reco Q).

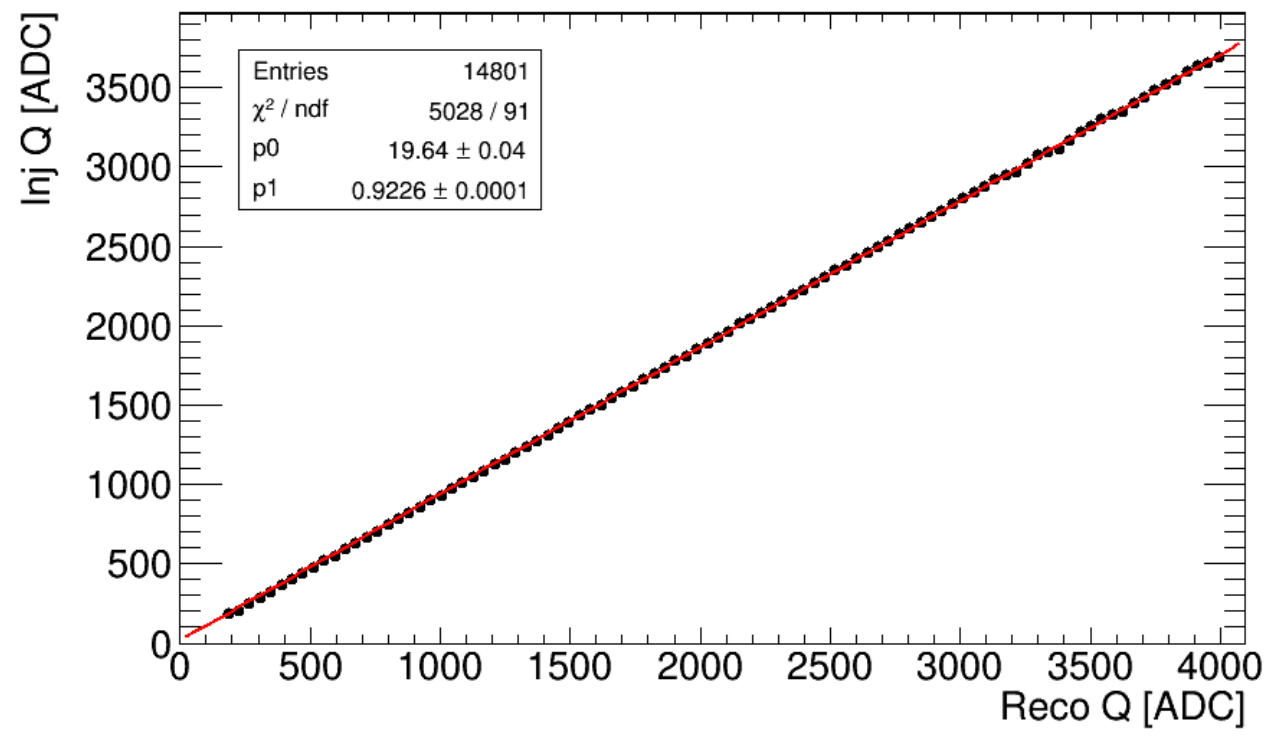

Figure 7. Profile histogram of the reconstructed charge as a function of the injected charge for a typical channel.

Related to the power consumption, the TilePPr prototype consumes around $30 \mathrm{~W}$ during operation. The consumption of the TilePPr is below the maximum allowable power consumption of $60 \mathrm{~W}$ specified in the AMC standard. 


\section{Conclusions}

The increased luminosity for the HL-LHC leads to the complete redesign of the front-end and back-end electronics of the TileCal. New readout architecture with full-digital trigger will be implemented to cope with the new requirements of the HL-LHC.

As part of the Demonstrator project, the first prototypes of the TilePPr has been designed and tested. The prototype presented in this note performs as expected, where jitter cleaners play a crucial role to achieve error free communications at data rates of $9.6 \mathrm{Gbps}$. The firmware has been carefully designed to optimize the use of FPGA resources, specifying the implementation of dedicated elements as block RAMs, clock buffers or PLLs when needed.

The TilePPr prototype will be used for the evaluation of the new readout architecture in the test-beam campaigns during 2015-2016 and for the operation of the new electronics which will be inserted in the ATLAS detector at the end of 2016.

\section{References}

[1] ATLAS collaboration, The ATLAS Experiment at the CERN Large Hadron Collider, 2008 JINST 3 S08003.

[2] ATLAS Collaboration, Readiness of the ATLAS Tile Calorimeter for LHC collisions, Eur. Phys. J. C, 70 (2010).

[3] ATLAS Collaboration, Letter of Intent for the Phase-II Upgrade of the ATLAS Experiment, CERNLHCC-2012-022, (2012).

[4] A. Valero et al., ATLAS TileCal Read-Out Driver production, 2007 JINST 2 P05003.

[5] S. Muschter et al., Development of a digital readout board for the ATLAS Tile Calorimeter upgrade demonstrator, 2014 JINST 9 C01001.

[6] F. Carrió et al., The sROD module for the ATLAS Tile Calorimeter Phase-II Upgrade Demonstrator, 2014 JINST 9 C02019.

[7] J. Anderson et al., FELIX: a High-Throughput Network Approach for Interfacing to Front End Electronics for ATLAS Upgrades, ATL-DAQ-PROC-2015-014, https://cds.cern.ch/record/2016626.

[8] PICMG, Advanced Mezzanine Card Base Specification, November 2006.

[9] Xilinx Corporation, http://www.xilinx.com.

[10] J. P. Cachemiche, Centre de Physique des Particules de Marseille, Module Management Controller mezzanine board - Specification", 2011.

[11] M. Barros et al., The GBT-FPGA core: features and challenges, 2015 JINST 10 C03021.

[12] T. Williams et al., IPbus A flexible Ethernet-based control system for xTCA hardware, 2014 JINST $10 \mathrm{C} 02019$ (2015). 